\title{
Use of colorimetric hydrogel as an indicator for food packaging applications
}

\author{
DUYGU ALPASLAN \\ Department of Chemical Engineering, Faculty of Engineering, Van Yuzuncu Yil University, Van 65080, Turkey \\ alpaslanduygu@gmail.com
}

MS received 10 October 2018; accepted 8 April 2019

\begin{abstract}
A novel hydrogel was synthesized from $N, N$-dimethyl acrylamide (DMAAm), gelatin, citric acid (CA) and Basilicum extract (BE). This study was aimed at creating an advanced food packaging material that allows the detection of food spoilage, which can be monitored colorimetrically. The poly(gelatin-co-DMAAm)/CA-BE was synthesized in the form of a film on Petri dishes using a redox polymerization technique. Mechanical and water resistance properties of the hydrogel were further improved by the addition of $\mathrm{CA}$ and $N, N$-methylene-bis-acrylamide as crosslinkers; $\mathrm{BE}$ was added to the reaction mixture and entrapped in the polymer chains with the aim to introduce antimicrobial, antioxidant and anthocyanin properties for the desired utilization. The characterization of the synthesized hydrogel was carried out using a dynamic and mechanical analyser, thermo gravimetric analyser, Fourier transform infrared spectroscopy and scanning electron microscopy. The antimicrobial activity of the hydrogel was observed as it was tested against Escherichia coli, Bacillus subtilis and Staphylococcus aureus. Furthermore, total antioxidant and anthocyanin activities of the hydrogels were also studied at different $\mathrm{pH}$ values to monitor the colour change capabilities. It was concluded that the hydrogel was a strong candidate for use in food packaging.
\end{abstract}

Keywords. Hydrogel; poly(gelatin-co-DMAAm)/CA-BE; antimicrobial effect; antioxidant activity; anthocyanin activity.

\section{Introduction}

The food sector has developed and expanded rapidly in the past couple of decades, which has also made it possible-and sometimes necessary - to transport food materials from one country or continent to another. The stability of the quality of the food material becomes an important challenge during such long-distance transportation. Instant monitoring of food quality is therefore vital for the controls at the borders, as well as for the convenience of the consumers in the market due to which smart food-packing materials have attracted notable attention from consumers, producers and controllers alike.

The basic function of food packaging is to protect the food from external and internal environmental conditions, like light, heat, pressure, moisture, microorganisms, vibration and gaseous emissions. Existing packaging applications, however, are unable to meet all these functions at once, and every type of packaging material or application has some sort of shortcoming. Intelligent food packaging may be a solution to these inadequacies in food packaging. These widely used intelligent food packages are sensitive to environmental factors, and inform the consumers by a change of colour which occurs due to mechanical, chemical, enzymatic or microbial deterioration. Food packaging materials are generally manufactured from glass, metals, plastics [1], papers [2], paperboards [3], polymers and fabric, and these are still commonly used in the food industry for the protection, security, convenience and containment of the food products. These materials also provide informative transmission, agglomeration and tamper indication capabilities. In recent years, polymeric materials like hydrogels have become the most commonly utilized materials for this purpose. Many natural materials, such as gelatin, starch and cellulose are also being used in the food packaging industry due to their biocompatibility, low toxicity and decomposability properties [4,5]. To improve their properties and introduce increased mechanical strength and antioxidant [6-8], antimicrobial $[9,10]$ and anthocyanin properties [11], additional natural compounds like citric acid (CA) [12-14] and pigments from Basilicum are sometimes added to the primary materials of the packaging. Furthermore, the addition of pigments gives these materials the ability to change colour, which occurs due to the $\mathrm{pH}$ shifts that occur during food deteriorations $[15,16]$.

Modifiable hydrogels, which are also named 'intelligent polymers', rapidly and reversibly respond to various physical and chemical conditions and stimuli, such as water, $\mathrm{pH}$, heat, UV light, daylight, electrostatic fields, magnetic fields and changes in physicochemical and microbiological properties. In recent decades, intelligent polymers have been used in the production of catalysts, adsorbents, food, wound dressings [17], drug-delivery systems, enriched media for microorganisms, cell culture substrates and modification 
agents of electrodes [18]. They have also been used in microbiology [19] and tissue engineering to obtain regenerative medicines [20-22].

The objective of this study is to synthesize, characterize and apply an intelligent hydrogel (poly(gelatin-coDMAAm)/CA-BE) with the addition of gelatin, CA and Basilicum extract (BE) as natural compounds, to obtain a novel instant food quality monitoring device that can be used as a food packaging material. The obtained hydrogel is poly (gelatin-co-DMAAm)/CA-BE, and it was synthesized in the form of a film on Petri dishes via redox polymerization of $N, N$-dimethyl acrylamide (DMAAm), gelatin, $\mathrm{CA}-$ and $\mathrm{BE}$. BE was added as the pigment source, thanks to which the colour of the poly(gelatin-co-DMAAm)/CA$\mathrm{BE}$ can change based on the changes in medium $\mathrm{pH}$ that may occur as a result of food decomposition. Morphological, surface, thermal and structural characterizations of the poly(gelatin-co-DMAAm)/CA-BE were carried out by scanning electron microscopy (SEM), thermogravimetric analyser (TGA) and Fourier transform infrared (FTIR) analyses. The swelling behaviour and $\mathrm{pH}$ sensitivity of the synthesized poly(gelatin-co-DMAAm)/CA-BE were also studied. Furthermore, the antimicrobial, antioxidant and colour-specific activities of poly(gelatin-co-DMAAm)/CA-BE were investigated. Escherichia coli, Bacillus subtilis and Staphylococcus aureus microorganisms were used to investigate the antimicrobial activities of poly(gelatin-co-DMAAm)/CA-BE.

\section{Materials and methods}

\subsection{Material}

DMAAm, gelatin (99\%), N,N-methylene-bis-acrylamide (MBA) $(99 \%)$, ethanol, sodium hydroxide $(\mathrm{NaOH})$ and $\mathrm{HCl}$ (36.5-38\% v/v) were purchased from Sigma; ammonium per sulphate (APS) (98\%) and $N, N, N, N$-tetramethylenediamine (TEMED) were purchased from Merck. All reagents were of analytical grade of the highest purity available and they were used without further purification. Basilicum was obtained from local suppliers. Distilled water (DI, $18.2 \mathrm{M} \Omega \mathrm{cm}$; Millipore Direct-Q3UV) was also used throughout this study. For antibacterial activity assays, three bacterial strains obtained from the Biology Department at Van Yüzüncü Y1l University were used. B. subtilis (ATCC 6633) and S. aureus (ATCC 6538) were utilized as the Gram-positive bacteria, while E. coli (ATCC 8739) was used as the Gram-negative bacteria.

\subsection{Solvent extraction from Ocimum basilicum}

For each type of hydrogel, $200 \mathrm{~g}$ Basilicum was ground, extracted with DI water and then concentrated with a rotary evaporator ( $30 \% \mathrm{v} / \mathrm{v}$ of $\mathrm{BE})$. The $\mathrm{BE}$ was stored at $4^{\circ} \mathrm{C}$ for further analyses, such as antioxidant and antimicrobial activities, and anthocyanin tests [15].

\subsection{Synthesis of hydrogels}

The redox polymerization technique was used for the preparation of poly(gelatin-co-DMAAm)/CA-BE hydrogels. To achieve this synthesis, $2 \mathrm{ml}$ of DMAAm was mixed with $2 \mathrm{~g}$ of gelatin in $5 \mathrm{ml}$ DI water, and then $5 \mathrm{ml}$ of BE and $0.1 \mathrm{~g}$ of $\mathrm{CA}$ were added to get entrapped in the polymer chains. Afterwards, MBA $(0.25 \mathrm{~mol} \%$ in proportion to the total monomer amount) was mixed through the vortex, followed by the addition of $60 \mu 1$ TEMED to the BE hydrogel mixture. Finally, the initiator solution APS ( $1 \mathrm{~mol} \%$ in proportion to the total monomer amount) in $100 \mu 1$ DI water was introduced. Following these steps, the solution was put into a plastic Petri dish with a $35 \mathrm{~mm}$ diameter and was allowed to polymerize and to complete crosslinking under ambient conditions. These preparation steps are schematically shown in figure 1 . The synthesized hydrogels were kept in DI water, which was renewed every $8 \mathrm{~h}$ for $24 \mathrm{~h}$ to eliminate unreacted monomers. Finally, hydrogels in the form of a film were dried in an oven at $40^{\circ} \mathrm{C}$ until their weights were stabilized, after which they were stored in $4^{\circ} \mathrm{C}$ for characterization and utilization.

\subsection{Swelling behaviour of hydrogels}

Swelling inspections of hydrogels were carried out in three runs at room temperature $\left(25^{\circ} \mathrm{C}\right)$ by placing certain amounts of dried hydrogels and poly(gelatin-co-DMAAm)/CA-BE in DI water. An increase in mass was periodically measured by weighing hydrogels and poly(gelatin-co-DMAAm)/CA-BE after blot-drying them with filter paper to remove the superficial water, and returning them to the same swelling media. Hydrogels were kept in swelling medium for $24 \mathrm{~h}$ to determine their maximum swelling value, $S_{\max } \%$.

The percent swelling $(S \%)$ degree as a function of time is calculated as

$$
S \%=\frac{M_{\mathrm{t}}-M_{0}}{M_{0}} \times 100,
$$

where $M_{0}$ and $M_{\mathrm{t}}$ are the initial mass and the mass of hydrogels at time $t$, respectively [19].

\subsection{FTIR spectra, TG and SEM analysis of hydrogels}

The thermal behaviour of the hydrogels and the poly(gelatinco-DMAAm)/CA-BE were determined using a Setaram Labsys Evo gravimetric analyser (TG/DSC 1600 model, France). Approximately 4-6 mg of sample was placed in a ceramic crucible and analysed during heating up to $50-1000^{\circ} \mathrm{C}$ under an argon atmosphere with a flow rate of $100 \mathrm{ml} \mathrm{min}^{-1}$ and at a heating rate of $10^{\circ} \mathrm{C} \mathrm{min}-1$. The FTIR analyses of DMAAm and gelatin-based hydrogels were conducted using FTIR, Thermo Nicolet iS10 FTIR spectrometer, USA) using ATR apparatus with a $4 \mathrm{~cm}^{-1}$ resolution between 4000 and $650 \mathrm{~cm}^{-1}$. SEM was performed to visualize the hydrogel pores of the composite hydrogels. The microstructure of the 


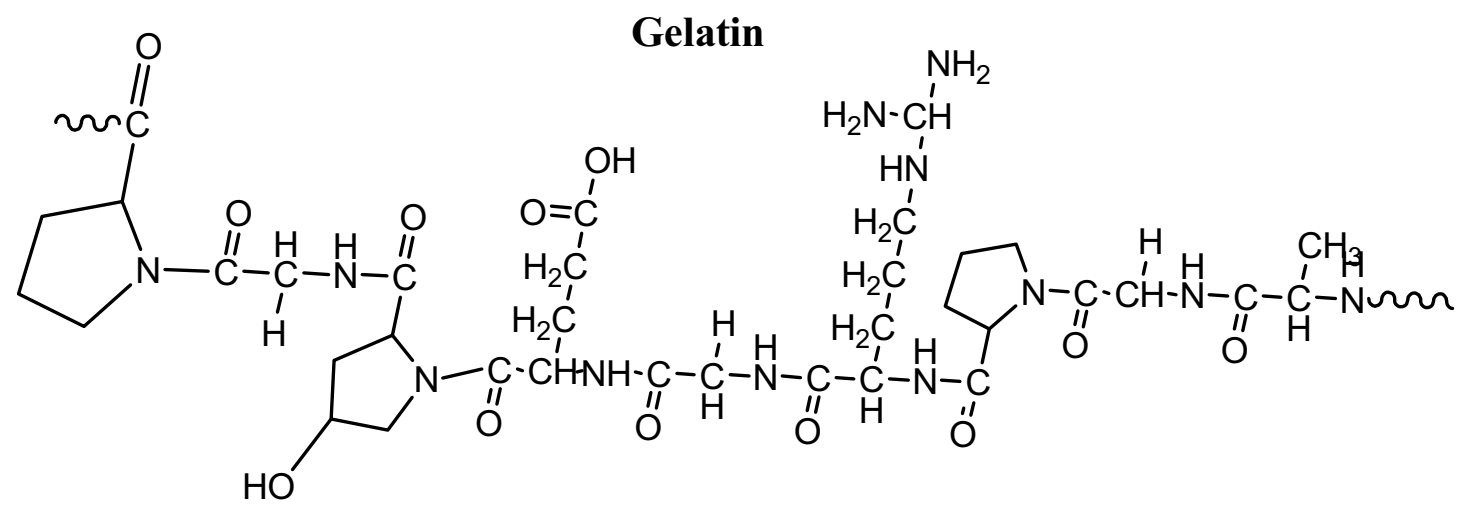

Citric acid (CA) N,N-Dimethylacrylamide (DMAAm)<smiles>O=C(O)CC(O)(CC(=O)O)C(=O)O</smiles><smiles>C=CC(=O)N(C)C</smiles>

\section{Basilicum extract}

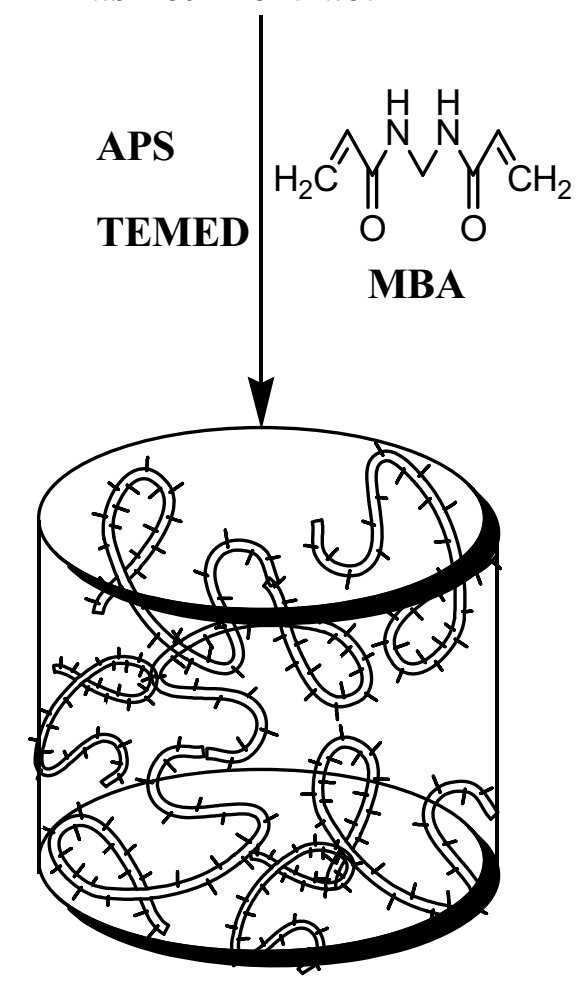

p(Gelatin-co-DMAAm)/CA-BE

Figure 1. Preparation of hydrogels. 
hydrogel was imaged using a SEM (Zeiss ultra field emission (FESEM-EDX), Germany).

\subsection{LC-MS/MS, Q Exactive analysis}

Liquid chromatograph mass spectrometer (LC-MS) (hybrid quadrupole-orbitrap mass spectrometer, Thermo, USA) was used to determine $\mathrm{BE}$ composition. The extract samples were renovated by capillary column Hypersil Gold $(50 \times 2.1 \mathrm{~mm})$, while helium was used as a carrier gas at a flow rate of $0.3 \mathrm{ml} \mathrm{min}{ }^{-1}$. The properties of individual components were determined by analytical standards (antimicrobial, antioxidant and anthocyanin).

\subsection{Mechanical strength test}

Dynamic and mechanical behaviours of the synthesized and modified materials were carried out using a dynamic mechanical analysis (DMA) (Mettler Tolledo Tritone Technology, UK) device. The samples were prepared in $\sim 1 \mathrm{~mm}$ height and $4 \mathrm{~mm}$ diameter. DMA measurements were recorded at a frequency of $1 \mathrm{~Hz}$ and at a heating rate of $5^{\circ} \mathrm{C} \mathrm{min}^{-1}$, while the temperature ranged from 30 to $80^{\circ} \mathrm{C}$. The modulus properties such as storage modulus and loss modulus were recorded as a function of temperature.

\subsection{Antimicrobial properties of hydrogels}

The agar dilution method was used to evaluate the antimicrobial activity as suggested in the literature $[19,23,24]$. $E$. coli (ATCC 8739), B. subtilis (ATCC 6633) and S. aureus (ATCC 6538) bacteria were used for the test. The test was run in three different media, i.e., the BE, poly(gelatin- co- $^{-}$ DMAAm)/CA and poly(gelatin-co-DMAAm)/CA-BE, and all were monitored for $24 \mathrm{~h}$. The number of bacteria were counted in cfu ml ${ }^{-1}[25,26]$.

\subsection{Antioxidant properties of hydrogels}

Folin-Ciocalteu and 2,2'-azino-bis(3-ethylbenzothiazoline6-sulphonic acid) (ABTS) methods were used to evaluate the antioxidant activity as explained in the literature [23].

\subsection{Anthocyanin properties of hydrogels}

The anthocyanin properties of hydrogels poly(gelatin-coDMAAm)/CA-BE, poly(gelatin-co-DMAAm), poly (gelatinco-DMAAm)/CA and $\mathrm{BE}$ were measured as indicated in the literature $[11,19,27]$. The absorbance of each dilution was then determined at $516 \mathrm{~nm}$ and the total absorbance of turbidity at $700 \mathrm{~nm}$. Anthocyanin activity was obtained from the maximum absorbance value by subtracting it from the total value. This procedure was repeated for each hydrogel.

\subsection{Colorimetric properties of hydrogels}

The effect of medium $\mathrm{pH}$ on poly(gelatin-co-DMAAm)/CA$\mathrm{BE}$ and the hydrogels was studied according to the CIEL $* a * b$ method [28]. Generally, the indicator is a weak acid (In H), which is in equilibrium with the surrounding $\mathrm{pH}$ according to the following equation:

$$
\text { In } \mathrm{H}_{k-I}^{k i}=\mathrm{In}^{-}+\mathrm{H}^{+} \text {, }
$$

where $\mathrm{H}_{k-I}^{k i}$ is the acidic colour intensity and $\mathrm{In}^{-}$is the alkali colour intensity. The variation of the medium colours with a variety of medium $\mathrm{pH}$ was measured within the visible region of the spectrum, while the poly(gelatin-co-DMAAm)/CA hydrogel was used as the blank sample.

The absorption spectra (380-770 $\mathrm{nm}$ ) of all solutions were recorded at constant intervals $(\Delta \lambda=2 \mathrm{~nm})$ with a spectrophotometer (Analytik jena, Specord S 600, Germany), using $5 \mathrm{~mm}$ path length quartz cells and distilled water as a reference. The CIELab parameters were calculated from the absorption spectra by using the original Croma Lab software, following the recommendations of the Commission International de L'Eclariage: CIE 1976 and the Standard Illuminant D65 $[29,30]$.

The $L^{*}$ defines lightness, $C^{*}$ specifies chroma and $h^{*}$ denotes the hue angle, which is an angular measurement.

$$
\begin{aligned}
& C^{*}=\left(a^{2}+b^{2}\right)^{1 / 2}, \\
& h=\arctan \left(\frac{b^{*}}{a^{*}}\right) .
\end{aligned}
$$

The CIELab parameters $\left(L^{*}, a^{*}, b^{*}, C^{*}\right.$ and $\left.h^{*}\right)$ were determined for $5 \mathrm{ml}$ solutions of each hydrogel at different $\mathrm{pH}$ values, ranging from 1 to $12 \Delta E$ (Euclidean). The values were calculated from the initial $\mathrm{pH}$ value $(\mathrm{pH} 1)$ and considering the Euclidean distance between the two colour points after each increase in $\mathrm{pH}$. The Euclidean distance of hydrogels was calculated with the following equation:

$$
\Delta E=\left(\left(\Delta L^{*}\right)^{2}+\left(\Delta a^{*}\right)^{2}+\left(\Delta b^{*}\right)^{2}\right)^{1 / 2} .
$$

Data consisted of an average of two experimental values.

$$
\begin{aligned}
& \Delta L^{*}=\text { difference in lightness/darkness value, } \\
& +=\text { lighter, }-=\text { darker, } \\
& \Delta a^{*}=\text { difference on red/green axis, } \\
& +=\text { redder, }-=\text { greener, } \\
& \Delta b^{*}=\text { difference on yellow/blue axis, } \\
& +=\text { yellower, }-=\text { bluer, } \\
& \Delta C^{*}=\text { difference in chroma, } \\
& +=\text { brighter },-=\text { duller }
\end{aligned}
$$


$\Delta H^{*}=$ difference in hue,

$\Delta E^{*}=$ total colour difference value.

\section{Results and discussion}

\subsection{Characterization of synthesized hydrogels}

The moisture content of a given food is one of the most important parameters for its shelf life and its structural degradation. It is known that polymers prepared from gelatin and DMAAm do not have good water resistance or mechanical strength, and are easily dissolved in aqueous media. Therefore, the water resistance and the mechanical strength of the $\mathrm{BE}$ was increased by the addition of CA to the polymer mixture during the synthesis as well as biocompatible MBA as a crosslinker [31-33].

In this context, intelligent hydrogels play an active role in determining the excess moisture in food packaging. Intelligent hydrogels in our study also absorbed excess water in the medium and their volume increased. Water resistance of the poly(gelatin-co-DMAAm)/CA-BE was found to be higher than that of poly(gelatin-co-DMAAm) and poly(gelatin-co-DMAAm)/CA in DI and at different $\mathrm{pH}$ values ranging from 2 to $12\left(25^{\circ} \mathrm{C}\right)$ as shown in figure 2. Caria et al [34] determined 670-700\% equilibrium swelling of poly(DMAAm).

As discussed before, it is known that gelatin hydrogels do not have strong water resistance properties. Thus, DMAAm was added to the gelatin hydrogels in our study to improve their hydrophobicity. Owing to the non-ionic nature of DMAAm in the hydrogel network, this does not dramatically change the swelling degree; in fact, the swelling degree was increased due to the presence of another

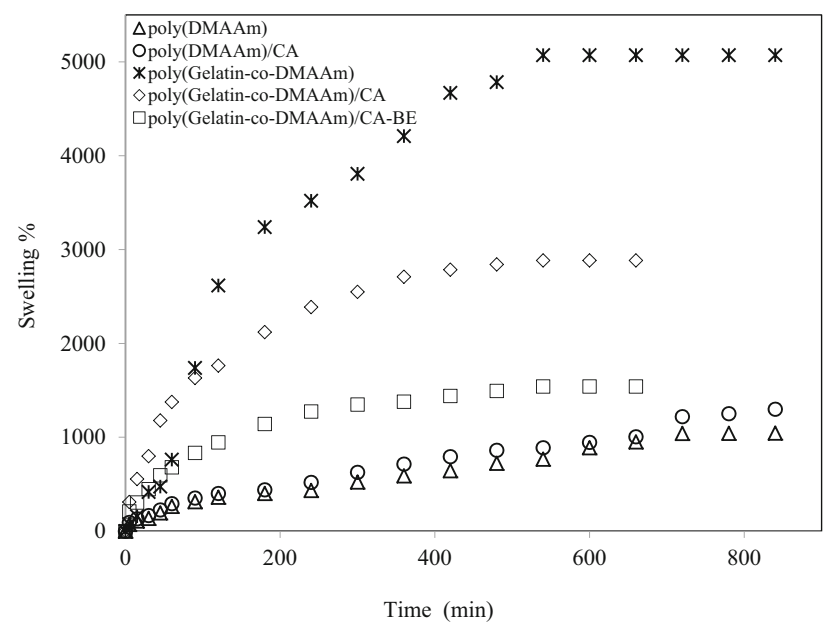

Figure 2. Percent swelling degree in hydrogels with time in DI water ( $S \%$ vs. time): poly(DMAAm), poly(DMAAm)/CA, poly(gelatin-co-DMAAm), poly(gelatin-co-DMAAm)/CA and poly(gelatin-co-DMAAm)/CA-BE.

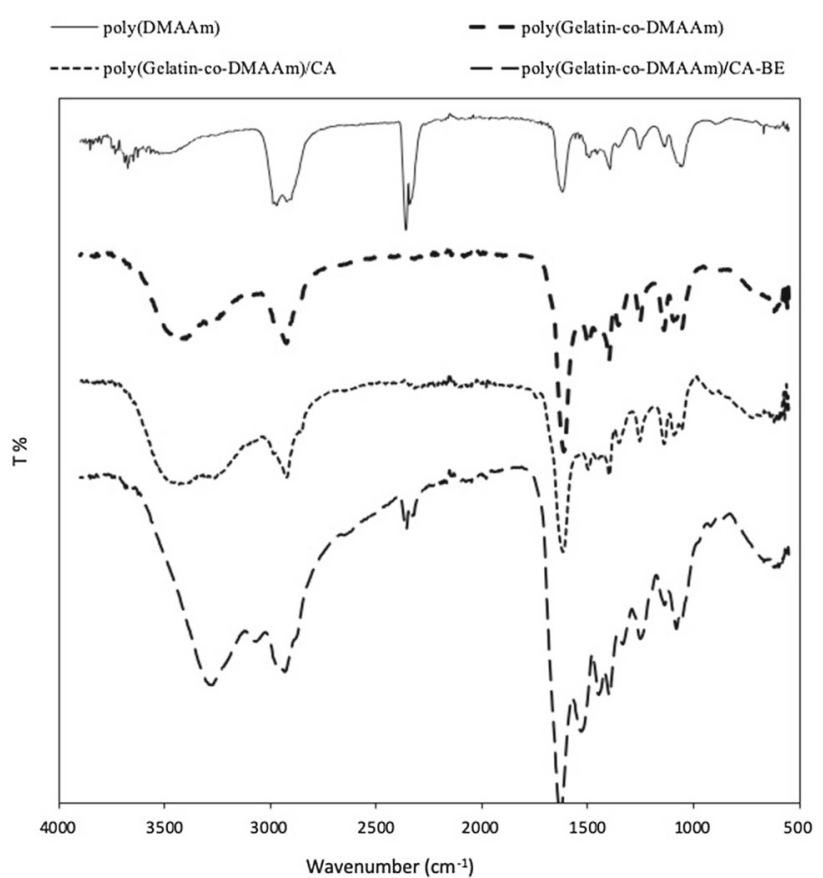

Figure 3. FTIR analysis of poly(DMAAm), poly(gelatin-coDMAAm), poly(gelatin-co-DMAAm)/CA and poly(gelatin-coDMAAm)/CA-BE.

monomer in the structure. When the swelling of poly(gelatinco-DMAAm)/CA-BE reached $1542 \%$, the water-swollen volume and area of hydrogels were increased by 14 and $4 \%$, respectively. The films prepared from poly(gelatinco-DMAAm)/CA-BE did not dissolve and were stable in aqueous media for more than 5 months. Relatively high waterabsorption of poly(gelatin-co-DMAAm)/CA-BE compared to the conventional food packaging materials enables it to respond rapidly to the $\mathrm{pH}$ changes in the media.

FTIR analysis utilizes the fundamental physics behind spectral wave absorption to obtain the molecular fingerprint of a given hydrogel. The FTIR spectra of poly(DMAAm) (figure 3) showed the characteristic peak belonging to the amide at $1616 \mathrm{~cm}^{-1}$, and the peaks between 1398 and $1250 \mathrm{~cm}^{-1}$ were related to $\mathrm{C}-\mathrm{N}$ stretching. Other peaks corresponding to the poly(gelatin-co-DMAAm)/CA hydrogel were missing, which can be justified by the new bonds established during the crosslinking process. When BE was added, the broad peak appearing between 1700 and $2500 \mathrm{~cm}^{-1}$ wavenumbers indicated that it formed new bonds, and the normally detected peaks between 1000 and $1500 \mathrm{~cm}^{-1}$ wavenumbers had disappeared. These appearances of new bonds and structural variances demonstrated the existence of a hydrogen-bonding interaction between poly(DMAAm), poly(gelatin-co-DMAAm)/CA and poly(gelatin-co-DMAAm)/CA-BE.

TGA measures the amount and speed of the change in the mass of a hydrogel as a function of temperature or time in a controlled atmosphere. Thermal stabilities of the synthesized 


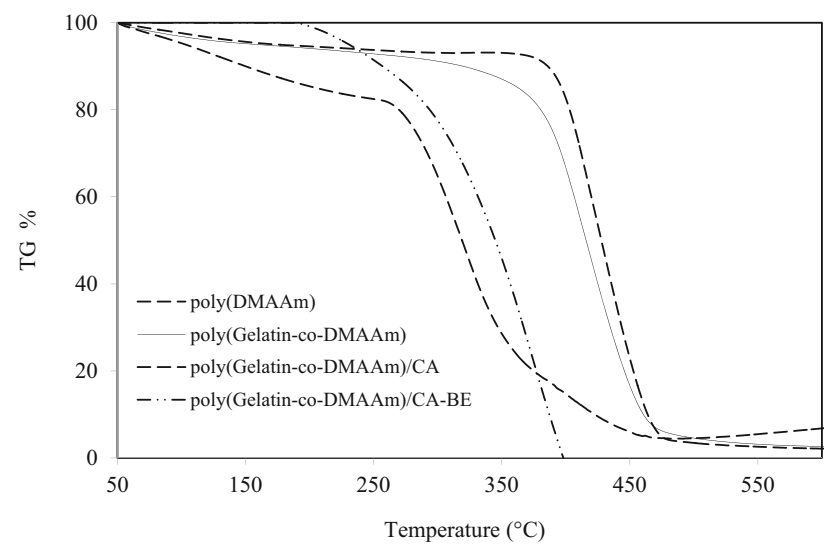

Figure 4. TGA of poly(DMAAm), poly(gelatin-co-DMAAm), poly(gelatin-co-DMAAm)/CA and poly(gelatin-co-DMAAm)/CABE.

hydrogels were investigated using a TGA/DSC analyser. In figure 4, the thermal degradation of the poly(DMAAm) hydrogel consisted of a single stage during which the total mass loss was about $97 \%$ when the temperature reached $500^{\circ} \mathrm{C}$. This can be explained by the accelerated mass loss of the hydrogel due to the main chains and cross-linked networks in poly(DMAAm) being destroyed [34]. Poly(gelatin-coDMAAm)/CA hydrogel degradation, on the other hand, had three stages. At the initial stage, the first mass loss of $\sim 15 \%$ $\left(205^{\circ} \mathrm{C}\right)$ was due to the evaporation of surface water and of the remaining small molecules. The second stage was between 250 and $359^{\circ} \mathrm{C}$, during which the mass loss reached $\sim 57.8 \%$. The third stage began at $359^{\circ} \mathrm{C}$ and the mass loss, which was about $19.8 \%$, tended to be constant until the temperature of $465^{\circ} \mathrm{C}$. Meanwhile, the thermal degradation of $\mathrm{BE}$ was observed in two steps as shown in figure 4 . The mass loss of $\mathrm{BE}$ at the first stage was $\sim 7.9 \%$ with the temperature range of $150-264^{\circ} \mathrm{C}$, and the hydrogel structure was drained completely off the surface water. The second step began when the temperature was in the range of $264-530^{\circ} \mathrm{C}$ and the average mass loss was $\sim 85 \%$. The total mass loss of poly(DMAAm-co-G)/CA-BE was achieved almost by $93.2 \%$ when the temperature rose up to $530^{\circ} \mathrm{C}$. It was observed that the number of degradation steps of the poly(gelatin-coDMAAm)/CA-BE hydrogel changed after the addition of BE to the structure of the hydrogel.

Surface morphologies of the synthesized materials were monitored using SEM, and the results are represented in figure $5 \mathrm{a}$ and $\mathrm{b}$. Figure 5a shows the surface image of the poly(gelatin-co-DMAAm)/CA hydrogel prepared with gelatin, DMAA and CA. As can be seen clearly, some roughness and some cracks and fractures were formed on the surface after polymerization. This image shows that the structure is neither tunable nor elastic. However, when BE was introduced into the polymerization reaction of the synthesized poly(gelatin-co-DMAAm)/CA-BE, cracks and fractures disappeared and the structure became porous and durable as shown in figure $5 \mathrm{~b}$. Some spherical-porous structures

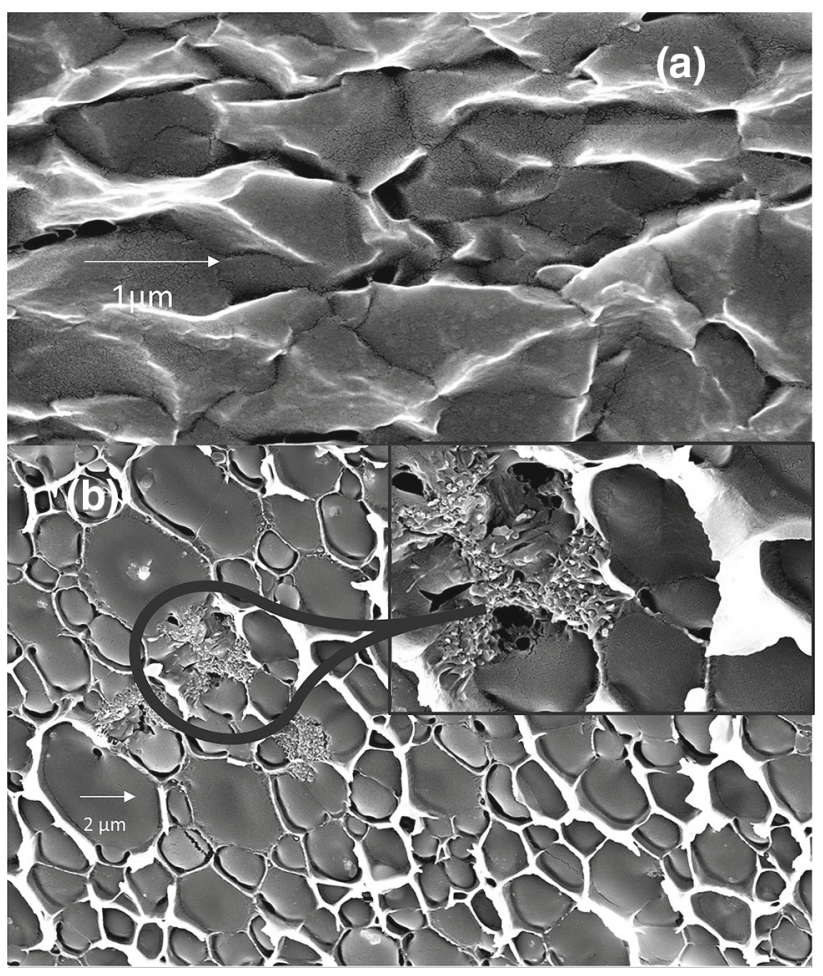

Figure 5. SEM images of poly(gelatin-co-DMAAm)/CA and poly(gelatin-co-DMAAm)/CA-BE.

were seen on the poly(gelatin-co-DMAAm)/CA-BE surface. Furthermore, after BE was added, it was verified by DMA analyses that poly(gelatin-co-DMAAm)/CA-BE was more durable than poly(gelatin-co-DMAAm)/CA.

\subsection{LC-MS/MS, $Q$ Exactive analysis}

Basilicum extracts include erulic acid, eriodictyol, quercetin, coumaric acid, vanillic acid and gallic acid, and all these extracts have antioxidant and antimicrobial activities. They also contain co-pigments and the substance known as pelargonidin, which has high anthocyanin activities [35, 36]. Co-pigmentation co-factors (co-pigments) consist of anthocyanin glucosides, phenolic acids, flavonoids and in particular, the derivatives of the flavonol and flavone subgroups [37,38].

To determine the presence of those substances, Basilicum was extracted with water and analysed using Q-Exactive (table 1). The detected compounds such as rutin, ferulic acid, eriodictyol, quercetin, coumaric acid, vanillic acid and gallic acid substances known for their antioxidant, antimicrobial and anthocyanin activities are found in Basilicum extracts. Furthermore, pelargonidin-3-O-(2" $-O$-glu $)$, which has even higher anthocyanin activities, was also detected $[7,39]$. These results indicate that rutin, ferulic acid, eriodictyol, quercetin, coumaric acid, vanillic acid and gallic acid and pelargonidin-3-O-(2" $-O$-glu $)$ contained in the extract might be providing certain synergistic and/or additive effects when mixed together. 
Table 1. Q-Exactive analysis of Basilicum (water extract) component.

List of Basilicum compounds

\begin{tabular}{|c|c|c|}
\hline Gallic acid & Naringenin-7-O-glucoside & Caffeoyltartaric acid \\
\hline Rutin & Pelargonidin-3-O-(2" -O-glu $)$ & Isorhamnetin-3-O-rutinoside \\
\hline Caffeic acid & Kaempferol-3-galactoside & cis-coutaric acid \\
\hline Eriodictyol & Coumaric acid hexoside & trans-coutaric acid \\
\hline Vanillic acid & Dihydroxycoumarin & Isorhamnetin-3-O-galactoside \\
\hline Fertaric acid & Quercetin hexoside isomer & Quercetin hexoside isomer \\
\hline Ferulic acid & 2-Quercetin-3-O-galactoside & Kaempferol-3-glucoside \\
\hline Naringenin & Quercetin-3-O-glucoside & 3-P-coumaroylquinic acid \\
\hline Kaempferol & Isorhamnetin & 4- $P$-coumaroylquinic acid \\
\hline Luteolin & Quercetin glucuronide & Quercetin-3- $O$-rutinoside \\
\hline
\end{tabular}

\subsection{Application of mechanical strength test}

The mechanical properties of hydrogels were studied by a DMA for their elasticity, and the results are shown in figure 6. Decreasing storage modulus values were found at low temperatures, which was interpreted as molecules moving easily due to the presence of more free volume [40]. It was also determined that poly(gelatin-co-DMAAm)/CA was more flexible and shapeable than the poly(gelatin-coDMAAm)/CA-BE, based on the decreasing storage modulus values. It was observed that the stored energy of poly(gelatinco-DMAAm)/CA increased between 40 and $60^{\circ} \mathrm{C}$ and that
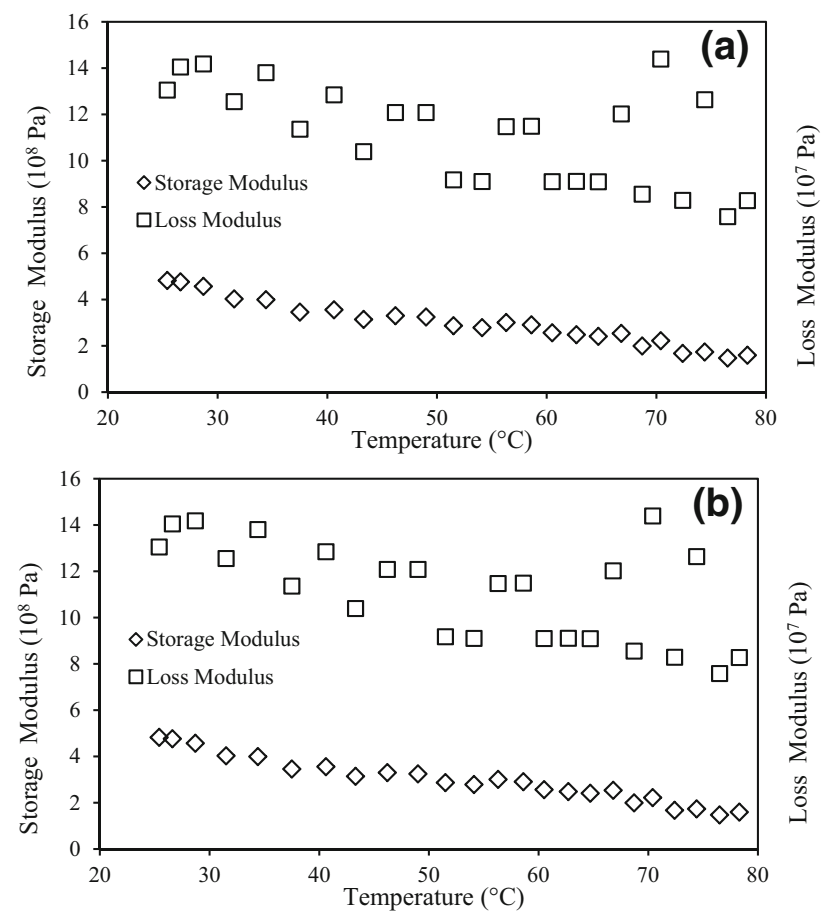

Figure 6. DMA analysis of (a) poly(gelatin-co-DMAAm)/CA and (b) poly(gelatin-co-DMAAm)/CA-BE. molecules were moving more in this temperature range (figure $6 \mathrm{a}$ ). In figure $6 \mathrm{~b}$, the energy storage of poly(gelatinco-DMAAm)/CA-BE can be seen to have decreased in the $20-80^{\circ} \mathrm{C}$ range, as the cross-linking property of hydrogel increases.

For the loss modulus, it was observed that poly(gelatin-coDMAAm)/CA-BE reduced the likelihood of losing energy. Sharp transitions in the loss storage curve indicated a high chemical resistance. Because of the poor mechanical strength of the gelatins, DMAAm, CA and MBA were added to the polymerization mixture to improve their mechanical strength and flexibility. Poly(gelatin-co-DMAAm)/CA possessed weaker mechanical properties compared to the poly(gelatin-co-DMAAm)/CA-BE. It was concluded that BE worked as a bridge molecule, which interacted with the DMAAm through hydrophilic interaction and promoted a better mixing with gelatin.

\subsection{Determination of antimicrobial properties of hydrogel}

The antimicrobial activity is an important feature that involves the production of antimicrobial substances, and/or the competitive dismissal of pathogen microorganisms. The antimicrobial activity of BE and poly(gelatin- $c o-D M A A m) / C A-B E$ was tested against three common bacteria and the obtained data are given in table 2. Poly(gelatin-co-DMAAm)/CA$\mathrm{BE}$ was treated with the bacteria for $72 \mathrm{~h}$, and the minimal inhibitory concentration (MIC) values were determined as $50 \mathrm{mg} \mathrm{ml}^{-1}$ against $S$. aureus and over $100 \mathrm{mg} \mathrm{ml}^{-1}$ against E. coli and B. cereus. The MBC values of BE, poly(gelatinco-DMAAm)/CA and BE were found to be over $150 \mathrm{mg} \mathrm{ml}^{-1}$ against $S$. aureus, E. coli and B. cereus. As presented in table 2 , the antimicrobial effects of the hydrogel are prominently strong compared to their counterparts in the literature [9,41-44]. The antimicrobial activity of BE is useful for food, agriculture and medicinal applications. The antimicrobial effect of poly(gelatin-co-DMAAm)/CA-BE will reduce the microbial count and/or inhibit the microbial growth in the environment when utilized properly. Many important 


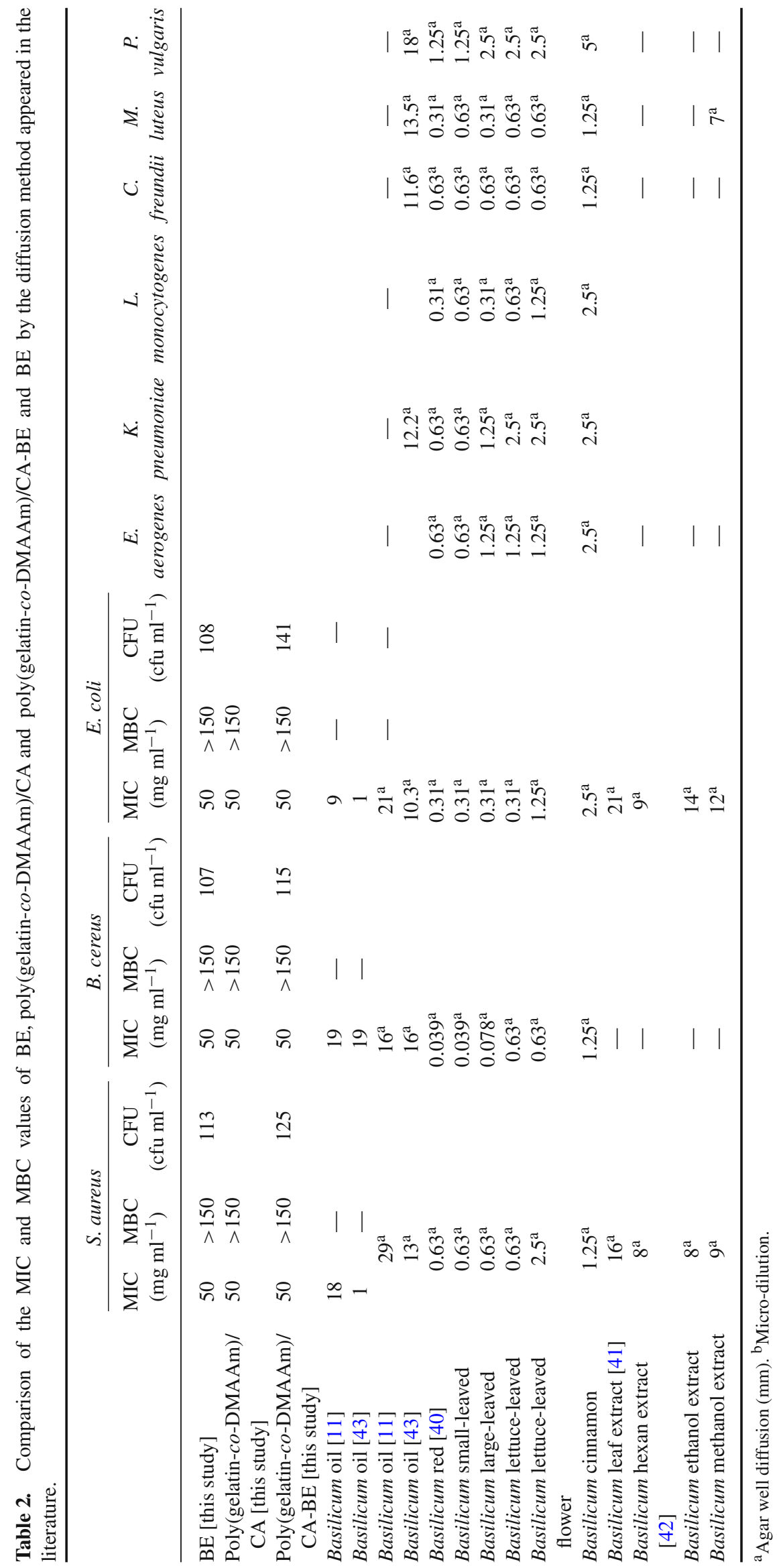


microbial and chemical deteriorative changes occur due to the reactions taking place within a given food. In addition to temperature, other environmental factors, such as water, colour and $\mathrm{pH}$ may induce deleterious changes that are catalysed by microbial growth in foods $[45,46]$. If microbial growth still occurs in the environment of poly(gelatin-co-DMAAm)/CA$\mathrm{BE}$, the change in the $\mathrm{pH}$ of the medium will be monitored by a colour change.

\subsection{Determination of antioxidant properties of hydrogels}

Antioxidants can neutralize free radicals (harmful molecules), preventing them from harming a living creature. As such, the antioxidant analysis was important for expected features of novel hydrogels. Total antioxidant activities of Basilicum, poly(gelatin-DMAAm)/CA and poly(gelatinDMAAm)/CA-BE of hydrogels were measured by ABTS and Trolox equivalent antioxidant capacity (TEAC) values. The gallic acid equivalent value of $\mathrm{BE}$, poly(gelatin-coDMAAm), poly(gelatin-co-DMAAm)/CA and poly(gelatinco-DMAAm)/CA-BE were calculated, and the results are displayed in table 3 . It was observed that the total antioxidant activities of BE and hydrogels were very well when compared to those given in the literature $[47,48]$. A significant proportion of the antioxidant activity originates from phenolic substances in the extract and the hydrogel structures, which were analysed in the Q-Exactive analysis. As a result, the antioxidant substances released from poly(gelatin-co-DMAAm)/CA-BE were determined to have the potential to minimize the negative effects of free radicals.

\subsection{Determination of anthocyanin properties of hydrogels}

As shown in table 4, total anthocyanin activities of $\mathrm{BE}$ and poly(gelatin-co-DMAAm)/CA-BE were determined for cyaniding-3-glucoside. It was concluded that the total anthocyanin activities of $\mathrm{BE}$ and the hydrogels were very promising when compared to their counterparts in the literature $[11,49]$. There are numerous physical and chemical factors which can have a negative impact on the stability of anthocyanin, the most important ones are increases or decreases in the temperature, light, oxygen concentration and $\mathrm{pH}$ levels, along with the presence or absence of ascorbic acid and metal ions $[50,51]$.

\subsection{Determination of colorimetric properties of hydrogels}

The colorimetric interpretation of co-pigmentation based on the CIELab colour diagram was demonstrated to be practical, since both the quantitative and the qualitative colour changes are understood more clearly. It was observed that $\mathrm{pH}$ and co-pigment structure and concentration had significant influences on the co-pigmentation process, which induced different absolute and relative colour changes in anthocyanin
Table 3. Comparison of total phenol content (gallic acid equivalent phenol content) and TEAC values of BE, poly(gelatin- $\mathrm{co}$ DMAAm), poly(gelatin-co-DMAAm)/CA, poly(gelatin-coDMAAm)/CA-BE and similar BE appeared in the literature.

\begin{tabular}{|c|c|c|}
\hline \multirow[b]{2}{*}{ Substance } & \multicolumn{2}{|c|}{ Antioxidant } \\
\hline & $\begin{array}{l}\text { Total phenol } \\
\quad\left(\mathrm{mg} \mathrm{g}^{-1}\right)\end{array}$ & $\begin{array}{c}\text { TEAC } \\
\left(\mu \mathrm{mol} \mathrm{g}^{-1}\right)\end{array}$ \\
\hline $\mathrm{BE}$ & 5724 & 2460 \\
\hline p(gelatin-co-DMAAm) & 93 & - \\
\hline $\mathrm{p}$ (gelatin-co-DMAAm)/CA & 310 & 1 \\
\hline Poly(gelatin-co-DMAAm)/ & 1824 & 275 \\
\hline \multicolumn{3}{|l|}{$\mathrm{CA}-\mathrm{BE}$} \\
\hline \multicolumn{3}{|l|}{ Basilicum type [47] } \\
\hline Cinnamon & 35.6 & 199 \\
\hline Dark opal & 126.2 & 547 \\
\hline Holy & 51.1 & 297 \\
\hline Italian large leaf & 62.9 & 354 \\
\hline Sweet dani lemon & 55.8 & 206 \\
\hline Osmin purple & 81.7 & 440 \\
\hline Purple ruffles & 92.6 & 497 \\
\hline Red rubin & 95.1 & 562 \\
\hline Sweet & 55.7 & 296 \\
\hline \multicolumn{3}{|l|}{ Basilicum origin [11-48] } \\
\hline Babol & 65.5 & 35.7 \\
\hline Birjand & 41.4 & 16.1 \\
\hline Brujerd & 41.5 & 19.8 \\
\hline Dezful & 23 & 10.8 \\
\hline Dezful & 29.5 & 12.9 \\
\hline Isfahan & 64.6 & 26 \\
\hline Isfahan & 44.9 & 19.8 \\
\hline Isfahan & 48.9 & 22.7 \\
\hline Kerman & 57.1 & 30.6 \\
\hline Kermanshah & 39.2 & 26.9 \\
\hline Khorram & 42.3 & 20.6 \\
\hline Khuzestan & 59.2 & 28.1 \\
\hline Mahallat & 63.8 & 35.2 \\
\hline Malavi & 42.9 & 19.7 \\
\hline Orumieh & 32.8 & 15.1 \\
\hline Qazvin & 38.6 & 19.1 \\
\hline Qom & 54.1 & 26.3 \\
\hline Sanandaj & 55.9 & 28.4 \\
\hline Shahr & 40.6 & 19.7 \\
\hline Yazd & 42.6 & 15.8 \\
\hline Zabol & 42.6 & 32.1 \\
\hline Zabol & 32.3 & 15 \\
\hline Zabol & 47.9 & 27.3 \\
\hline Dark opal & 126.2 & 547 \\
\hline Holy & 51.1 & 297 \\
\hline Italian large leaf & 62.9 & 354 \\
\hline Sweet dani lemon & 55.8 & 206 \\
\hline Osmin purple & 81.7 & 440 \\
\hline Purple ruffles & 92.6 & 497 \\
\hline Red rubin & 95.1 & 562 \\
\hline Sweet & 55.7 & 296 \\
\hline
\end{tabular}

solutions [52-54]. Table 5 represents the chromatic characteristics of poly(gelatin-co-DMAAm)/CA-BE according 
Table 4. Comparison of anthocyanin values of BE, poly(gelatinco-DMAAm)/CA-BE and Basilicum appeared in the literature.

\begin{tabular}{lc}
\hline & Anthocyanin $\left(\mathrm{mg} \mathrm{ml}^{-1} \mathrm{mg} \mathrm{g}^{-1}\right)$ \\
\cline { 2 - 2 } Substance & Cyanidin-3-glucoside \\
\hline BE [this study] & 0.4 \\
Poly(gelatin-co-DMAAm)/CA-BE & 0.9 \\
Basilicum type [11] & \\
Dark opal & 0.19 \\
Holy scared red & 0.13 \\
Opal & 0.13 \\
Osmin purple & 0.12 \\
Purple bush & 0.18 \\
Purple ruffles & 0.19 \\
Red rubin & 0.17 \\
Red rubin purple leaf & 0.18 \\
Rubin & 0.17 \\
Basilicum type [50] & \\
Dark opal & 0.19 \\
Holy scared red & 0.09 \\
Opal & 0.13 \\
Osmin purple & 0.19 \\
Purple bush & 0.07 \\
Purple ruffles & 0.19 \\
Red rubin & 0.17 \\
Red rubin purple leaf & 0.18 \\
Rubin & 0.18 \\
\hline
\end{tabular}

Table 5. Colour parameters (CIELab) $L^{*}, a^{*}$ and $b^{*}$ measured for poly(gelatin-co-DMAAm)/CA-BE in different $\mathrm{pH}$ solutions.

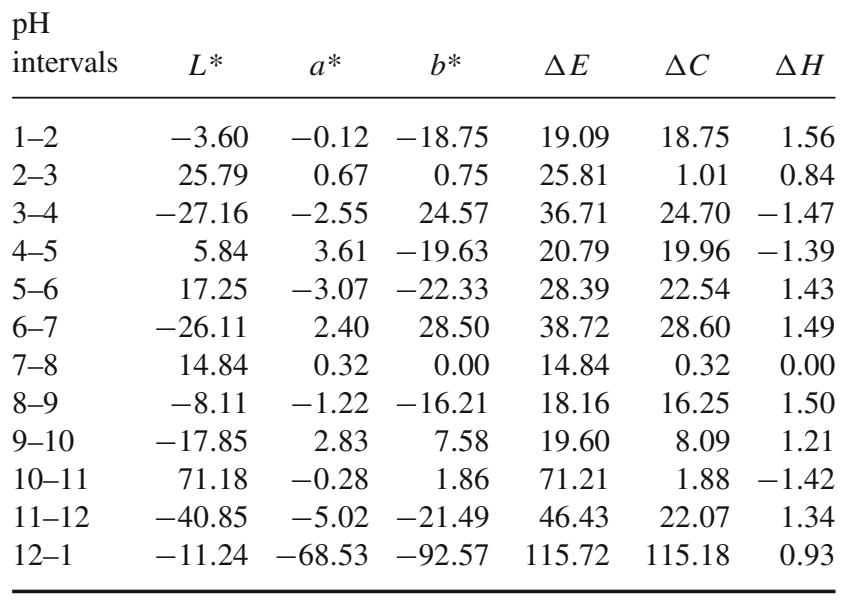

to the CIELab colour diagram $[29,30]$. Each colour has its own distinct appearance, based on three elements: hue $(a)$, chroma $(b)$ and value (lightness) $(L)[16,55]$. The colour change of poly(gelatin-co-DMAAm)/CA-BE was measured by immersing in water for $5 \mathrm{~min}$, in which the $\mathrm{pH}$ values ranged from 1 to 12 . A visible colour change was detected instantly on the poly (gelatin-co-DMAAm)/CA-BE after contact with water. The colorimetric parameters $\Delta L^{*}, \Delta a^{*}$ and $\Delta b^{*}$ for poly(gelatin-co-DMAAm)/CA-BE were calculated using equation (5) with varying $\mathrm{pH}$ values from 1 to 12 .
$\Delta E^{*}$ was calculated to be 115.72 . It is reported that about 3-unit change in $\Delta E^{*}$ could be easily detected by the average human eye [54]. The $a^{*}$ value of -68.53 indicates greener or less red colour, while the $b^{*}$ value of -92.57 indicates yellow or less blue colour. As clearly indicated in table 5, as $\mathrm{pH}$ moves from 1 to $12, \Delta L^{*}, \Delta C^{*}$ and $\Delta H^{*}$ values were calculated to be $115.72,115.18$ and 0.93 , respectively. This means that poly(gelatin-co-DMAAm)/CA-BE at $\mathrm{pH} 1$ is lighter in colour than poly(gelatin-co-DMAAm)/CA-BE at $\mathrm{pH}$ 12. BE was also utilized by Demirhan and Özbek [51] in their study where they measured the colour of the dry basil and reported a final total colour change of $\Delta E^{*} 2.973$ for all Basilicum [51]. The values of the difference between colour forms which were observed due to changes in $\mathrm{pH}$ were also distinctly high. It was concluded that poly(gelatin-co-DMAAm)/CA-BE easily responded to $\mathrm{pH}$ changes caused by chemical and/or microbiological activities in the media.

It is difficult to determine the $\mathrm{pH}$ changes and inhibition effect on microorganisms existing in a given food [56-58]. The strong antimicrobial, antioxidant, anthocyanin and copigmentation (the distinguishable colour change) properties of $\mathrm{BE}$ at different $\mathrm{pH}$ levels contribute to the poly (gelatin-coDMAAm)/CA-BE to enable the preservation of food. Moreover, the poly(gelatin-co-DMAAm)/CA-BE could widely be used in kinds of food materials like milk, meat, vegetables and fruits. Many microorganisms in the spoilage of milk, meat, vegetables and fruits could be inhibited by poly(gelatin-coDMAAm)/CA-BE, and the colour shifting can be observed if these products were to spoil. We believe that the results of this study demonstrate that poly(gelatin-co-DMAAm)/CA$\mathrm{BE}$ may function as a quality indicator and preservative for various food products.

\section{Conclusion}

The poly(gelatin-co-DMAAm)/CA-BE, which was synthesized through the redox polymerization technique from DMAAm, gelatin, $\mathrm{CA}$ and $\mathrm{BE}$, is a biocompatible and intelligent polymer. It could thus be used as a non-toxic component for food packaging applications and as a safety indicator. The poly(gelatin-co-DMAAm)/CA-BE has a high potential for use in increasing the lifetime of foods on the shelves. The antimicrobial and antioxidant properties of the poly(gelatin-co-DMAAm)/CA-BE make it valuable as an alternative solution in the struggle against pathogen microorganisms and free radicals in food packaging, food preservation and biomedical materials. Significant colour shifts based on the change of medium $\mathrm{pH}$ were observed on the synthesized poly(gelatin-co-DMAAm)/CA-BE, which is caused by its inherent anthocyanin properties. We hope that the poly(gelatin-co-DMAAm)/CA-BE will be one of the main means of establishing traceability systems for effective and sustainable food safety. 


\section{References}

[1] Marsh K and Bugusu B 2007 J. Food Sci. 7239

[2] Biji K B, Ravishankar C N, Mohan C O and Gopal T K S 2015 J. Food Sci. Technol. 526125

[3] Mahalik N P 2009 Sens. Instrum. Food Qual. Safe. 312

[4] Kathuria N, Tripathi A, Kar K K and Kumar A 2009 Acta Biomater. 5406

[5] Bot A, Amerongen I, Groot R D, Hoekstra N L and Agterof W G M 1996 Polym. Gels Netw. 434

[6] Leal P F, Maia N B, Carmello Q A C, Catharino R R, Eberlin M N and Meireles M A A 2007 Food Bioprocess Technol. 1 326

[7] Lee S-J, Umano K, Shibamoto T and Lee K-G 2005 Food Chem. 91131

[8] Pedro A C, Moreira F, Granato D and Rosso N D 2016 An. Acad. Bras. Cienc. 881055

[9] Moghaddam A M D, Shayegh J, Mikaili P and Sharaf J D 2011 J. Med. Plants Res. 53

[10] Rattanachaikunsopon P and Phumkhachorn P 2010 Biosci. Biotechnol. Biochem. 741200

[11] Phippen W B 1999 Anthocyanin instability in basil (Ocimum basilicum L.) (Doctoral, Purdue University, USA)

[12] Ghanbarzadeh B, Almasi H and Entezami A A 2011 Ind. Crops Prod. 33229

[13] Rocha-García D, Guerra-Contreras A, Reyes-Hernández J and Palestino G 2017 Eur. Polym. J. 8942

[14] Christian D, Roberta D S, Francesca S, Alessandro S, Giuseppe V, Alfonso M et al 2008 J. Appl. Polym. Sci. 1102453

[15] Abou-Arab A A, Abu-Salem F M and Abou-Arab E A $2011 \mathrm{~J}$. Am. Sci. 711

[16] Bobelyn E, Hertog M L A T M and Nicolaï B M 2006 Postharvest Biol. Technol. 42104

[17] Sahiner M, Alpaslan D and Bitlisli B O 2014 Polym. Bull. 71 16

[18] Sahiner N and Alpaslan D 2014 J. Appl. Polym. Sci. 13110

[19] Alpaslan D, Sahiner M, Yuceer Y, Akcali A, Aktas N and Sahiner N 2016 Polym. Bull. 733351

[20] Galaev I Y and Mattiasson B 1999 Trends Biotechnol. 175

[21] Qing G, Lu Q, Xiong Y, Zhang L, Wang H, Li X et al 2017 Adv. Mater. 291604670

[22] Yin P 2012 Hydrogel-based nanocomposites and laserassisted surface modification for biomedical application. Postdoctoral, The University of Western Ontario London, Ontario, Canada

[23] Yayintas O T, Alpaslan D, Yuceer Y K, Yilmaz S and Sahiner N 2016 Nat. Prod. Res. 182169

[24] Duygu Alpaslan, Tuba Erşen Dudu and Aktaş N 2018 MANAS J. Eng. 618

[25] Childs O E and Bardsley W G 1975 Biochem. J. 14510

[26] Singleton V L and Rossi J A 1965 A. J. Enol. Vitic. 1612

[27] Brouillard R and Delaporte B 1977 J. Am. Chem. Soc. 998

[28] Wrolstad R E, Durst R W and Lee J 2005 Trends Food Sci. Technol. 16423

[29] Torskangerpoll K and Andersen Ø M 2005 Food Chem. 89 427
[30] Walkowiak-Tomczak D and Czapski J 2007 Food Chem. 104 709

[31] Lee K Y, Rowley J A, Eiselt P, Moy E M, Bouhadir K H and Mooney D J 2000 Macromolecules 334

[32] Reddy N and Yang Y J 2010 Food Chem. 118702

[33] Menzel C 2014 Starch structures and their usefulness in the production of packaging materials (PhD doctoral, Swedish University, Uppsala)

[34] Caria G, Alzari V, Monticelli O, Nuvoli D, Kenny J M and Mariani A 2009 J. Polym. Sci. A: Polym. Chem. 471422

[35] Bylka W, Matlawska I and Pilewski N A 2004 Jana 79

[36] Xiao J B, Ren F L and Xu M 2006 J. Pharma. Allied Sci. 33

[37] Boulton R 2001 Am. J. Enol. Vitic. 5210

[38] Baranac J M, Petranovic N A and Dimitric-Markovic J M 1996 J. Agric. Food Chem. 443

[39] Jayasinghe C, Gotoh N, Aoki T and Wada S 2003 J. Agric. Food Chem. $\mathbf{5 1} 7$

[40] Menard K P 1999 Dynamic mechanical analysis: a practical introduction (Boca Raton, Florida, USA: CRC Press LLC)

[41] Eriotou E, Anastasiadou K, Nikolopoulos D and Koulougliotis D 2015 J. Nutr. Food Sci. 51

[42] Khalil A 2013 Biotechnology 121

[43] Adıgüzel A, Güllüce M, Şengül M, Öğütücü H, Şahin F and Karaman İ 2005 Turk. J. Biol. 295

[44] Gaio I, Saggiorato A G, Treichel H, Cichoski A J, Astolfi V, Cardoso R I et al 2015 J. Consum. Prot. Food Safety 106

[45] Kilcast D and Subramaniam P 2000 The stability and shelf life of food (Boca Raton, Cambridge, England: CRC Press Woodhead Pub)

[46] in’tVeld J H J H 1996 I. J. Food Microbiol. 3318

[47] Juliani H R and Simon J E 2002 Antioxidant activity of basil (Alexandria, Virginia, USA: vol 575 ASHS Press)

[48] Javanmardi J, Stushnoff C, Locke E and Vivanco J M 2003 Food Chem. 833

[49] Simon J E, Morales M R, Phippen W B, Vieira R F and Hao Z 1999 Perspect. New Cops New Uses 16499

[50] Giusti M M and Wrolstad R E 2001 in: Current protocols in food analytical chemistry M M Giusti and R E Wrolstad (eds) (Hoboken: John Wiley and Sons, Inc.) F1.2.1

[51] Demirhan E and Özbek B 2009 Dry. Technol. 27156

[52] Gordillo B, Rodriguez-Pulido F J, Escudero-Gilete M L, Gonzalez-Miret M L and Heredia F J 2012 J. Agric. Food Chem. 602896

[53] Gordillo B, Cejudo-Bastante M J, Rodriguez-Pulido F J, JaraPalacio M J, Ramirez-Perez P, Gonzalez-Miret M L et al 2014 J. Agric. Food Chem. 622663

[54] Martínez J A, Melgosa M, Pérez M M, Hita E and Negueruela A I 2001 Revista Agaroquimica Tecno. Alimentos 75

[55] Heredia F J, Francia-Aricha E M, Rivas-Gonzalo J C, Vicario I M and Santos-Buelga C 1998 Food Chem. 63491

[56] Lee O H and Lee B Y 2010 Bioresour. Technol. 1013751

[57] Yoshida C M P, Maciel V B V, Mendonça M E D and Franco T T 2014 LWT_Food Sci. Technol. 5583

[58] Hurtado N H, Morales A L, González-Miret M L, EscuderoGilete M L and Heredia F J 2009 Food Chem. 11788 